\title{
Measuring gamma doses over the mGy-kGy range with a single type of TLD detector
}

\author{
Adrien Gruel ${ }^{1}$, Alix Sardet ${ }^{1}$, Vincent Chaussonnet ${ }^{1}$, Maxime Houdouin-Quenault ${ }^{1}$ and Daniel Garnier ${ }^{1}$ \\ ${ }^{1}$ CEA, DES, IRESNE, DER, Cadarache F-13108 Saint-Paul-Lez-Durance, France \\ adrien.gruel@cea.fr
}

\begin{abstract}
Thermo-luminescent detectors are currently used to measure gamma doses in the medical and environmental surveillance fields. During the past few years, the CEA Reactor Studies Division tested and validated the use of these detectors for gamma flux characterization and nuclear heating measurements in mixed neutron/gamma fields of low power reactors. Doses were comprised between a few mGy and a few Gy for dose rates up to a few $G y \cdot h^{-1}$. However, in MTR or TRIGA reactors, the gamma flux level is much higher $\left(>10^{12} \mathrm{n} / \mathrm{cm}^{2} / \mathrm{s}\right)$ and the TLD currently in use $\left(\mathrm{CaF}_{2}: \mathrm{Mn}\right.$ and ${ }^{7} \mathrm{LiF}: \mathrm{Mg}$, $\left.\mathrm{Ti}\right)$ and their readout protocols were no longer suitable for the resulting doses. In order to extend the applicable dose range up to $1 \mathrm{MGy}$ (dose rate of a few $k G y \cdot h^{-1}$ ), several options were explored. On one side, some adjustments were made to the readout protocols of $\mathrm{CaF}_{2}: \mathrm{Mn}$ and ${ }^{7} \mathrm{LiF}: \mathrm{Mg}, \mathrm{Ti}$, notably by testing the use of filters to reduce the amount of light received by the reader PMT to avoid saturation. On the other side, a new type of TLD ( $\mathrm{LiF}: \mathrm{Mg}, \mathrm{Cu}, \mathrm{P}$ ) with different $\mathrm{Li}$ enrichments (natural or enriched in ${ }^{7} \mathbf{L i}$ ) was tested.

This paper presents the calibration measurements results performed in pure gamma fields, at the irradiation platform of the CEA Cadarache Radioprotection Division, between $250 \mathrm{mGy}$ and $3 \mathrm{~Gy}$ for all detector types. In addition to the calibration, these measurements also studied the $\mathrm{Mg}, \mathrm{Cu}, \mathrm{P}$ doped detectors response: reproducibility, dose rate dependence, incoming photon energy dependence, high temperature effect when reading TLD, etc.

Results show that at low doses Mg,Cu,P-doped TLDs are slightly less stable than $\mathrm{CaF}_{2}: \mathrm{Mn}$ and ${ }^{7} \mathrm{LiF}: \mathrm{Mg}$,Ti. The sensitivity modification after a high dose exposure seems to indicate that a new protocol readout should be defined for $\mathrm{Mg}, \mathrm{Cu}, \mathrm{P}$-doped sensors (high temperature peak).
\end{abstract}

Keywords - TLD, calibration, gamma dose.

\section{INTRODUCTION}

$\mathrm{T}$ HERMO-LUMINESCENT detectors are mainly used to measure gamma doses in the medical and environmental surveillance fields. These detectors can also be used for nuclear heating measurements in the mixed neutron/gamma field of low power reactors. To characterize doses and nuclear heating in Material Testing Reactors, their range of use should be extended to be able to reach kGy or even MGy. This would enable the use of a single type of detector to carry out measurements in various locations, from the core periphery to the outermost components.
In this work we compare the performances of four types of thermoluminescent detectors:

$$
\begin{array}{ll}
\text { - } & \mathrm{CaF}_{2}: \mathrm{Mn}, \text { (hereafter named TLD400) } \\
\text { - } & { }^{7} \mathrm{LiF}: \mathrm{Mg}, \mathrm{Ti} \text { (hereafter named TLD700) } \\
\text { - } & { }^{7} \mathrm{LiF}: \mathrm{Mg}, \mathrm{Cu}, \mathrm{P} \text { (hereafter named MCP7) } \\
\text { - } & { }^{\text {nat }} \mathrm{LiF}: \mathrm{Mg}, \mathrm{Cu}, \mathrm{P} \text { (hereafter named MCPN) }
\end{array}
$$

The two first types of sensors are well characterized in the field of low dose measurements ( $\lesssim 1 \mathrm{~Gy}$ ) in zero power experimental reactors [1]-[5]. They were also tested in the JSI's TRIGA reactor at doses up to $1 \mathrm{kGy}$ [6], where TLD400 showed a good agreement against calculations. The two last types are LiF-based detectors but with different doping elements, that should increase their sensitivities, as well are make them suitable for high dose measurements [7][8]. They are labelled $\mathrm{MCP}$ in the following.

During irradiations, the mm-sized detectors are set in cylindrical aluminium pillboxes with $2-\mathrm{mm}$ wall thickness to ensure the charged particle equilibrium (Fig. 1).
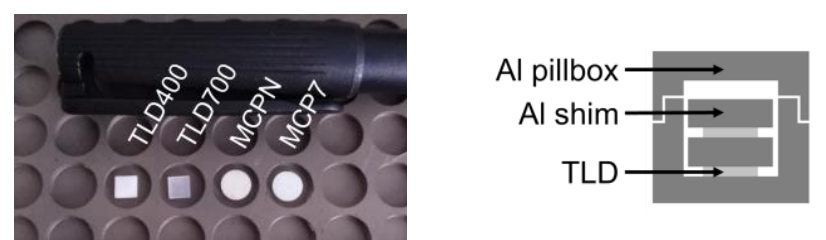

Fig. 1. Left: TLDs. Right: simplified schematics of the sensors in an aluminium pillbox.

The reader system is a Harshaw 3500. For each sensor, two temperature profiles for the readout phase were used. The first is the one recommended by the constructor. The second one is a test law, in order to emphasize possible modifications in the luminescence curves after the irradiations. Readout parameters are presented in Table I and illustrated in Fig. 2.

30 of each MCP-type TLDs were irradiated, 5 of which were read with the test law. 10 TLD400 and 10 TLD700 were irradiated, half of them being read with a test law.

For TLD400 and TLD700, annealing protocol consists in heating detectors at $400{ }^{\circ} \mathrm{C}$ for 1 hour and $100{ }^{\circ} \mathrm{C}$ for 2 hours. For MCP7 and MCPN, it consists of heating the detectors at $240{ }^{\circ} \mathrm{C}$ for $10 \mathrm{~min}$.

Tests of those sensors at doses up to 3 Gy are presented in the second section of this work, while preliminary tests after a high dose exposure at $50 \mathrm{kGy}$ are presented in the third part. 
TABLE I

READOUT TEMPERATURE PROFILE PARAMETERS. BOLD VALUES EMPHASIZE DIFFERENCES AGAINST REFERENCE PARAMETERS

\begin{tabular}{ccccc}
\hline \hline & $\begin{array}{c}\text { Starting } \\
\text { temperature } \\
\left({ }^{\circ} \mathrm{C}\right)\end{array}$ & $\begin{array}{c}\text { Temperature } \\
\text { Increase Rate } \\
\left({ }^{\circ} \mathrm{C} . \mathrm{s}-1\right)\end{array}$ & $\begin{array}{c}\text { Maximum } \\
\text { tempereature } \\
\left({ }^{\circ} \mathrm{C}\right)\end{array}$ & $\begin{array}{c}\text { Total time } \\
(\mathrm{s})\end{array}$ \\
\hline TLD400 & 150 & 10 & 350 & 30 \\
TLD400 test & 150 & $\mathbf{2 5}$ & $\mathbf{3 7 0}$ & 30 \\
\hline TLD700 & 140 & 15 & 280 & 23.3 \\
TLD700 test & 140 & $\mathbf{1 0}$ & 280 & 23.3 \\
\hline MCP & 140 & 15 & 240 & 30 \\
MCP test & 140 & 15 & $\mathbf{5 0 0}$ & 30 \\
\hline \hline
\end{tabular}

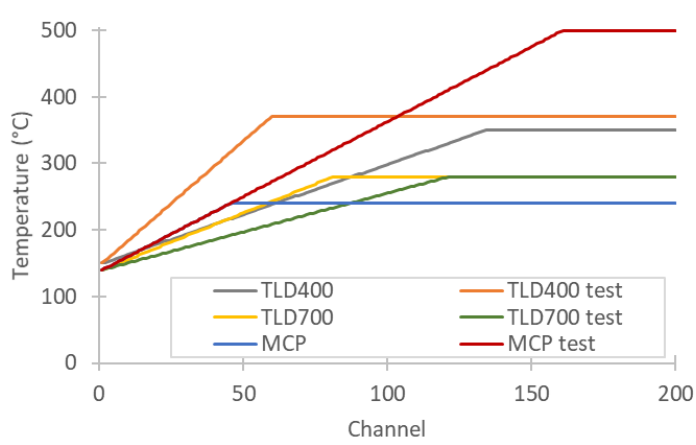

Fig. 2. Temperature profiles.

\section{DETECTORS PERFORMANCE COMPARISON FOR LOW DOSES MEASUREMENTS}

In this section, performance of different types of TLDs are studied against various parameters, at doses up to $3 \mathrm{~Gy}$. Measurements were carried out at CEA Cadarache Radioprotection Division's irradiation platform. Several ${ }^{60} \mathrm{Co}$ and ${ }^{137} \mathrm{Cs}$ gamma sources are available, each one calibrated against the Henri Becquerel National Laboratory, a reference institute in the field of radiation measurements. On this platform, the detectors position can be set at any distance from 80 to $500 \mathrm{~cm}$; and irradiation time is set to the second. The highest dose rate reachable is around $300 \mathrm{mGy} \cdot \mathrm{h}^{-1}\left({ }^{60} \mathrm{Co}\right.$ source), with an uncertainty below $1 \%$ at one standard deviation.

First, reproducibility studies are presented, then dose rate and gamma energy dependences, and finally linearity against integrated dose.

\section{A. Reproducibility of the measurements}

The aim of this study is to characterize the stability of the TLDs' response for a given experimental setup. Namely, the type of source, the source-detector distance (meaning the dose rate), the total integrated dose and the readout and annealing protocols are kept constant. Four irradiations at $500 \mathrm{mGy}$ at a distance of $80 \mathrm{~cm}$ of a ${ }^{60} \mathrm{Co}$ are used to assess the sensors' reproducibility. The reproducibility over $n$ measurements is evaluated using:

$$
U_{x}=\frac{1}{\sqrt{n}} \sqrt{\frac{1}{n-1} * \sum_{i=1}^{n}\left(x_{i}-\bar{x}\right)^{2}},
$$

$x_{i}$ being the integrated charge of the $i^{\text {th }}$ measurement during the readout process, and $\bar{x}$ the average of the $n$ measurements.

With the standard readout protocol, TLD400 and TLD700 show a very good reproducibility: $3.4 \%$ and $4.8 \%$, respectively, consistent with past studies [3]. The test law worsen this reproducibility, especially for TLD700: $4.5 \%$ and $7.0 \%$, respectively.

In this dose range, the reproducibility of the $\mathrm{Mg}, \mathrm{Cu}, \mathrm{P}$-doped detectors is not as good as TLD400 and TLD700. With the recommended readout protocol, it is $9.8 \%$ for MCPN and $5.8 \%$ for MCP7. With the test law (heating up to $500{ }^{\circ} \mathrm{C}$ ), the results deteriorate very significantly, the reproducibility being higher than $20 \%$ for both types. Indeed, the luminescence curves could be very different from an irradiation to another. This effect cannot be attributed to irradiation conditions, since all detectors are irradiated simultaneously.

\section{B. Response dependence against dose rate and photon energy}

To test dose rate dependence of the TLDs' responses, irradiations targeting at the same total integrated dose were carried out, the distance between the source and the detectors being modified between $80 \mathrm{~cm}$ to $140 \mathrm{~cm}$. Irradiations characteristics are presented in Table II, two doses were investigated: $250 \mathrm{mGy}$ and $750 \mathrm{mGy}$.

TABLE II

CHARACTERISTICS OF IRRADIATIONS FOR DOSE-RATE INFLUENCE

\begin{tabular}{cccc}
\hline Total dose & Distance $(\mathrm{cm})$ & Dose rate $\left(\mathrm{mGy}^{-1}\right)$ & Irradiation time (s) \\
\multirow{3}{*}{$250 \mathrm{mGy}$} & 80 & $330 \pm 0.7 \%$ & 2727 \\
& 100 & $211 \pm 0.65 \%$ & 4265 \\
$750 \mathrm{mGy}$ & 140 & $107 \pm 0.6 \%$ & 8411 \\
& 80 & $327 \pm 0.7 \%$ & 8257 \\
& 100 & $211 \pm 0.65 \%$ & 12796 \\
\hline \multirow{3}{*}{70} & $107 \pm 0.6 \%$ & 25234 \\
\hline
\end{tabular}

For TLD400 and TLD700 the discrepancies between the integrated charges at different dose rates are below or of the order of magnitude of the reproducibility. From those irradiations, we can conclude that these sensors are not sensitive to the dose rate, at least in the tested range.

MCPN and MCP7 showed a good behavior for irradiations carried out at $750 \mathrm{mGy}$, the discrepancies between the integrated charges at different dose rates being below or of the order of magnitude of the reproducibility. Results after irradiation at $250 \mathrm{mGy}$ are less consistent. Luminescence curves could be very different from an irradiation to another. It is therefore more difficult to conclude. Nevertheless, it seems that $\mathrm{Mg}, \mathrm{Cu}, \mathrm{P}$-doped TLDs are less stable for low doses.

The gamma energy dependence of the detectors' response has been tested by comparing ${ }^{137} \mathrm{Cs}(662 \mathrm{keV})$ and ${ }^{60} \mathrm{Co}$ (1173 keV and $1332 \mathrm{keV}$ ) irradiations at the same dose level. In this study, all tested detectors and all readout protocols proved to be stable. Discrepancies between irradiations are of the same order of magnitude as the reproducibility (see previous section). It should however be noted that only two irradiations were carried out (one with ${ }^{137} \mathrm{Cs}$ and one with ${ }^{60} \mathrm{Co}$ ), and that a more comprehensive study could bring interesting additional new results by studying lower and higher gamma energies.

\section{Response linearity}

In this section, individual calibration curves for each detector are obtained with irradiations between 200 mGy and 3000 mGy 
for TLD400 and TLD700, and between $200 \mathrm{mGy}$ and $1000 \mathrm{mGy}$ for MCPN and MCP7. Indeed, measurements of $\mathrm{Mg}, \mathrm{Cu}, \mathrm{P}$-doped sensors above $1000 \mathrm{mGy}$ lead to saturation of the TLD reader.

It is assumed that the detectors' response, meaning the integrated charge obtained as the integral of the luminescence curve, is linear versus dose.

For TLD400 and TLD700 the final uncertainty on the calibration factor is below $2 \%$ on average. The dispersion of the calibration factor values is around $5 \%$ for TLD400 and $7 \%$ for TLD700. As this is slightly higher than the reproducibility observed, it is mandatory to carry out individual sensor calibration, unless having more homogeneous sensor batches. It should be noted that the dispersion is smaller using the recommended readout protocol.

For $\mathrm{Mg}, \mathrm{Cu}, \mathrm{P}$-doped detectors, uncertainties on the calibration factor are slightly higher, 5.6\% and $3.6 \%$ for MCPN and MCP7, respectively. The dispersion of the calibration factor values is consistent with that of TLD400 and TLD700: around $5 \%$ for MCPN and $6 \%$ for MCP7. Results are far less satisfactory with the test law (max. temperature of $500{ }^{\circ} \mathrm{C}$ ): uncertainties are between $16 \%$ to $20 \%$ and dispersion around $15 \%$.

On this dose range, TLD700 are less sensitive (16 nC. $\mathrm{mGy}^{-1}$ on average) than TLD400, which are three times more sensitive (43 nC. $\mathrm{mGy}^{-1}$ on average). $\mathrm{Mg}, \mathrm{Cu}-\mathrm{P}$-doped TLDs are about five times more sensitive than TLD400, with an average sensitivity of $200 \mathrm{nC} . \mathrm{mGy}^{-1}$ for $\mathrm{MCPN}$ and $230 \mathrm{nC} . \mathrm{mGy}^{-1}$ for MCP7.

Examples of luminescence curves obtained for calibration purposes for a TLD400 and for a MCP7 are presented Fig. 3 and Fig. 4.

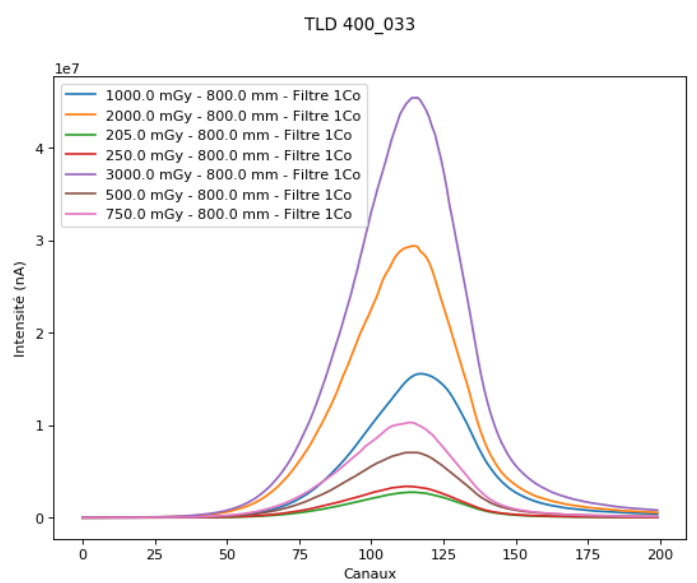

Fig. 3. Luminescence curves at different doses for a TLD400 after irradiations carried out for the calibration phase ("Filtre 1Co": measurement with 100\%transmittance filter, irradiation in front of a cobalt source).

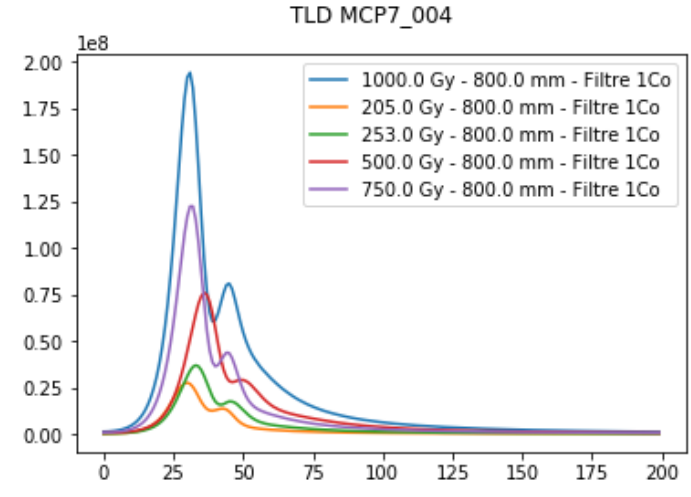

Fig. 4. Luminescence curves at different doses for a MCP7 after irradiations carried out for the calibration phase. ("Filtre 1Co": measurement with 100\%transmittance filter, irradiation in front of a cobalt source)

\section{BEHAVIOR AFTER HIGH DOSE EXPOSURE}

Detectors were exposed to a $50 \mathrm{kGy}$ dose at the EPICUR irradiation facility, operated by the IRSN (French Institute for Radiation Protection and Nuclear Safety). This facility is devoted to physicochemical studies on confined iodine under irradiation. It consists of a small irradiation chamber (about $30 \mathrm{~cm}$ in width, $30 \mathrm{~cm}$ in length and $60 \mathrm{~cm}$ in height) with intense ${ }^{60} \mathrm{Co}$ sources, where the dose rate ranges from $0.1 \mathrm{kGy} \cdot \mathrm{h}^{-1}$ to $20 \mathrm{kGy} \cdot \mathrm{h}^{-1}$ given the irradiation position in the cavity.

For this first test, detectors were subjected to a $50 \mathrm{kGy}$ irradiation, and they were split in two batches with different dose rates. In each batch, two detectors of each type were irradiated.

Reference dose rates were measured with alanine dosimetry, with a typical uncertainty of $10 \%$. Several alanine dosimeters were set around void aluminium pillboxes in each position, to catch the average dose rate inside pillboxes. The highest dose rate was $8.2 \pm 0.2 \mathrm{kGy} \cdot \mathrm{h}^{-1}$, the lowest was $1.81 \pm 0.04 \mathrm{kGy} \cdot \mathrm{h}^{-1}$. The uncertainty corresponds to the discrepancy of the reference dosimetry for each irradiation location.

The sensitivity modification of the sensors after this high dose irradiation was verified with a $750 \mathrm{mGy}$ irradiation on the CEA Cadarache Radioprotection Division's irradiation platform. The evolution of luminescence curves obtained for a $750 \mathrm{mGy}$ irradiation before and after the $50 \mathrm{kGy}$ exposure are shown in Fig. 5 to Fig. 7.

There is a large sensitivity increase for TLD400: the total integrated charge was multiplied by a factor between 4 and 6.6. For TLD700 the sensitivity slightly decreased, by a factor about 1.5. For those two types of TLDs, the luminescence curve shape was not impacted by the high dose exposure. The temperature profile used (constructor or test) does not seem to have an impact on the sensitivity change before and after the high dose exposure

For MCPN and MCP7 sensors, two cases appear regarding the readout process:

- With the constructor law (maximum temperature of $240{ }^{\circ} \mathrm{C}$, top graphs of Fig. 6 and Fig. 7), the glow curve shape cannot really be analyzed anymore, since no peak 
can be distinguished. It seems that the temperature is not high enough to release the trapped electrons, and therefore to be able to read the integrated dose.

- With the test law (maximum temperature of $500{ }^{\circ} \mathrm{C}$, bottom graphs of Fig. 6 and Fig. 7), a peak at higher temperature appears, while the intensities of the peaks at $200{ }^{\circ} \mathrm{C}$ and $300{ }^{\circ} \mathrm{C}$ decreased by an order of magnitude. Their overall sensitivity (total integrated charge) decreased by a factor between 2.5 and 3 for MCPN, and by a factor between 4.5 and 5 for MCP7. With this test law, it has been verified that the high temperature peak was not due to the signal caused by the heating of the plate nor of the TLD itself. For comparison, this signal is plotted in yellow dashed lines on the bottom graphs in Fig. 6 and 7, and represent only a small fraction of the high temperature signal.
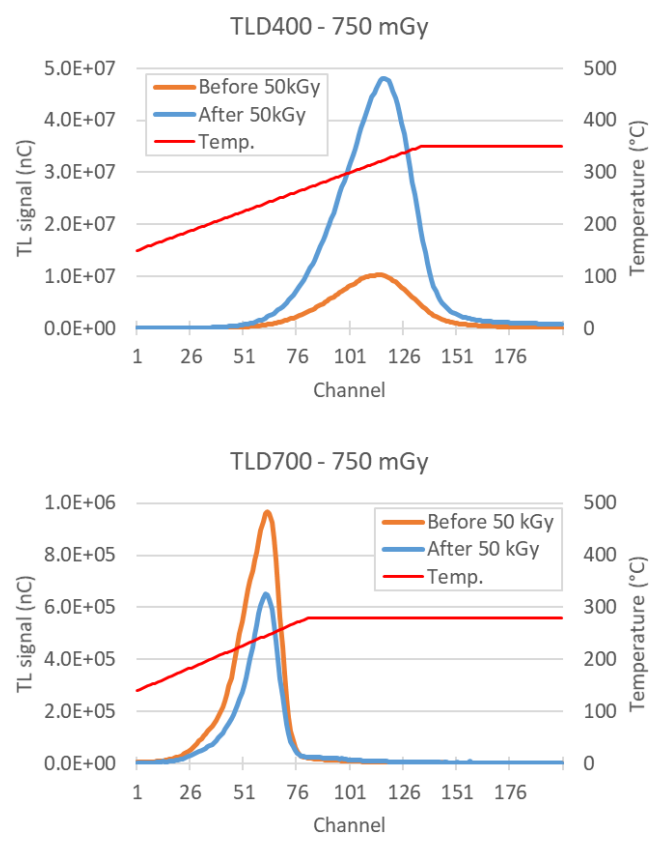

Fig. 5. Luminescence curves for TLD400 and TLD700 before and after $50 \mathrm{kGy}$ exposure. Measurement after a $750 \mathrm{mGy}$ irradiation in front of a ${ }^{60} \mathrm{Co}$ source with the standard readout protocol.
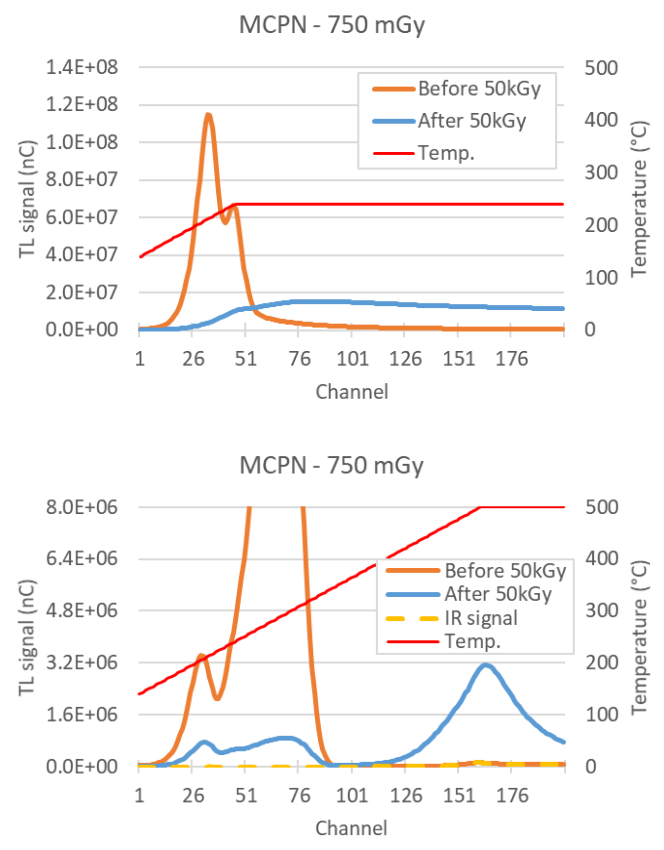

Fig. 6. Luminescence curves MCPN TLDs before and after $50 \mathrm{kGy}$ exposure. Measurement after a $750 \mathrm{mGy}$ irradiation in front of a ${ }^{60} \mathrm{Co}$ source. Top: constructor readout protocol, bottom: test readout protocol.
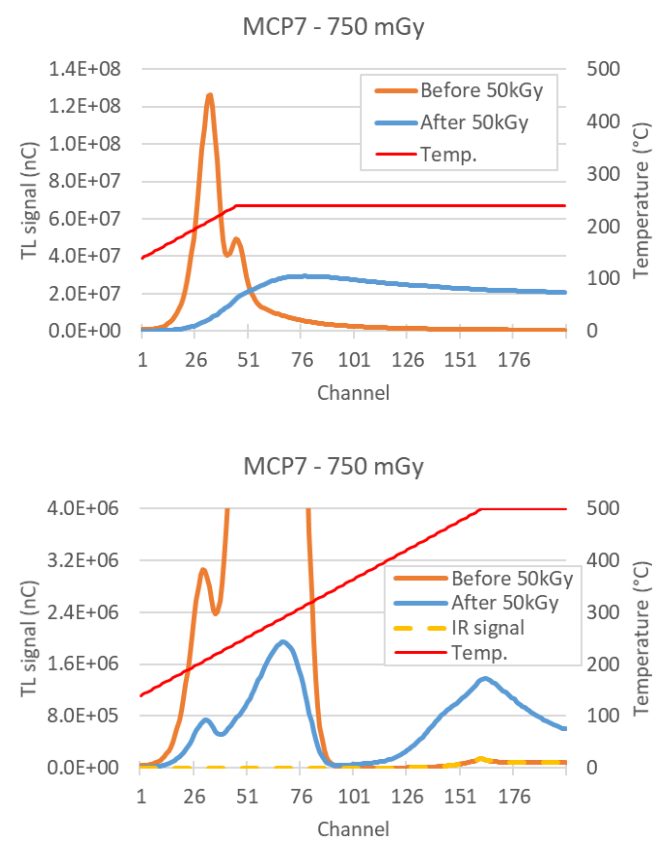

Fig. 7. Luminescence curves MCP7 TLDs before and after $50 \mathrm{kGy}$ exposure. Measurement after a $750 \mathrm{mGy}$ irradiation in front of a ${ }^{60} \mathrm{Co}$ source Top: constructor readout protocol, bottom: test readout protocol.

\section{CONCLUSIONS}

This work presents the study of different TLDs behavior regarding reproducibility, dose rate influence and gamma energy dependence.

In the low dose range ( $<3$ Gy) TLD400 and TLD700 seem to present better characteristics. Indeed, reproducibility is higher for $\mathrm{Mg}, \mathrm{Cu}, \mathrm{P}$-doped sensors. As the luminescence curves 
are less stable regarding irradiations conditions, individual calibration factors present higher final uncertainties for those detectors.

Sensitivity change was preliminary characterized, and different behaviors were described. This sensitivity change seemingly being definitive with the annealing protocol used, a new calibration for highly irradiated sensors should be carried out. High-dose irradiation for calibration up to several hundred of $\mathrm{kGy}$ should also be performed.

After high dose calibration, TLD detectors will be used to characterize the gamma field and nuclear heating in the TRIGA Mark II reactor at the Jožef Stefan Institute (JSI) in Slovenia. Measurements are expected to take place in the first half of 2022, in the frame of a CEA-JSI collaboration dedicated to the benchmark of the TRIGA reactor neutron and photon 3D transport model.

The large amount of data available will also be used to determine material physical properties to model TLDs luminescence curves.

\section{ACKNOWLEDGMENT}

The authors wish to thank the SPR/LRIR EDEPE group for the calibration phase at their irradiation platform.

\section{REFERENCES}

[1] M. Le Guillou, et al., State of the art on nuclear heating measurement methods and expected improvements in zero power research reactors, EPJ Nuclear Sci. Technol., vol. 3, p. 11, 2017.

[2] H. Amharrak, et al., Analysis and recent advances in gamma heating measurements in MINERVE facility by using TLD and OSLD techniques, IEEE Transactions on Nuclear Science, vol. 59, no. 4, pp. 1360-1368, Aug. 2012.

[3] H. Amharrak, Développement et optimisation de méthodes de mesures d'échauffements nucléaires et de flux gamma dans les réacteurs expérimentaux : identification, maîtrise, traitement et réduction des incertitudes associées, thesis, Aix-Marseille University, 2012.

[4] H. Amharrak et al., Development and Optimization of Nuclear Heating Measurement Techniques in Zero Power Experimental Reactors, IEEE Transactions on Nuclear Science, vol. 61, no. 5, pp. 2515-2526, Oct. 2014.

[5] M. Lemaire, Validation des calculs d'échauffements photoniques en réacteur d'irradiation au moyen du programme experimental AMMON et $d u$ dispositive CARMEN, thesis, Aix-Marseille University, 2015

[6] A. Gruel, et al., Gamma-heating and gamma flux measurements in the JSI TRIGA reactor, results and prospects, IEEE Transactions on Nuclear Science, 67 (4), pp. 559-567.

[7] B. Obryk, et al., Method of thermoluminescent measurement of radiation doses from micrograys up to a megagray with a single LiF:Mg,Cu,P detector, Radiat Prot Dosimetry, vol. 144, no. 1-4, pp. 543-547, Mar. 2011

[8] B. Obryk, et al., High-dose TL dosimetry of reactor neutrons, Radiat Prot Dosimetry, vol. 180, no. 1-4, pp. 235-239, Nov. 2017. 